\title{
Comparación clínica entre pacientes con déficit selectivo de inmunoglobulina A y otras inmunodeficiencias primarias
}

\author{
Clinical comparison between patients with selective immunoglobulin A \\ deficiency and other primary immunodeficiencies
}

\author{
Dra. Natalia A. Lozano ${ }^{a}$ Dr. Alejandro Lozano ${ }^{a}$, Dra. Laura V. Sasia ${ }^{a}$, Dr. Ricardo J. Saranz ${ }^{a}$, \\ Dra. María Fernanda Agresta ${ }^{a}$ Dra. María del Pilar Bovina Martijena ${ }^{a}$ Dr. Luciano Ianiero ${ }^{a}$ y \\ Dr. Andrés R. Grenat ${ }^{a}$
}

\begin{abstract}
RESUMEN
Las inmunodeficiencias primarias (IDP) son enfermedades de baja prevalencia. Existen signos de alarma para su sospecha clínica.

Los objetivos fueron describir características clínicas y signos de alarma de pacientes con IDP y comparar diferencias clínicas entre déficit selectivo de inmunoglobulina A (IgA) y otras IDP. Se estudiaron 89 pacientes, con mediana de edad, al momento del diagnóstico, de 6 años (4,08-11,67). 53 (59,5\%) pacientes, varones. $54(60,7 \%)$ pacientes, con déficit selectivo de IgA, y $35(39,3 \%)$, con otras IDP. Las principales manifestaciones clínicas fueron rinofaringitis en $65(73,03 \%)$ pacientes y atopia en $39(43,82 \%)$ pacientes. Presentaron signos de alarma $24(26,97 \%)$ pacientes, ninguno con déficit selectivo de IgA. En los pacientes con otras IDP, se observó mayor incidencia de infección respiratoria baja, sepsis, infecciones cutáneas, candidiasis mucocutánea, alteraciones dentales, malformaciones cardiovasculares, angioedema, internaciones y mortalidad. $10(28,57 \%)$ pacientes recibieron gammaglobulina endovenosa; 15 (42,85\%), antibiótico-profilaxis; y 2 (2,24\%), antimicótico-profilaxis.

Palabras clave: infecciones respiratorias, hipersensibilidad, gammaglobulinas, sindromes de inmunodeficiencia, deficiencia de IgA.
\end{abstract}

http:/ /dx.doi.org/10.5546/aap.2015.141

\section{INTRODUCCIÓN}

Las inmunodeficiencias primarias (IDP) son un grupo heterogéneo de enfermedades, vinculadas a más de 200 defectos genéticos que afectan al sistema inmune. ${ }^{1,2}$

a. Servicio de Alergia e Inmunología, Clínica Universitaria Reina Fabiola. Cátedra de Inmunología, Facultad de Medicina, Universidad Católica de Córdoba, Argentina.

Correspondencia:

Dra. Natalia A. Lozano: nalozano@yahoo.com

Financiamiento:

Clínica Universitaria Reina Fabiola. Facultad de Medicina,

Universidad Católica de Córdoba.

Conflicto de intereses: Ninguno que declarar.

Recibido: 13-6-2014

Aceptado: 21-11-2014
La prevalencia de estas enfermedades es difícil de establecer por las diferencias regionales y la heterogeneidad clínica y de laboratorio de las distintas patologías. Son enfermedades de baja prevalencia (de 1/10 000 a 1/500 000 nacidos vivos). ${ }^{1,3-5}$ Las IDP más frecuentes son las inmunodeficiencias de anticuerpos $\mathrm{y}$, dentro de estas, el déficit selectivo de inmunoglobulina A ( $\operatorname{Ig} \mathrm{A})$, que presenta una prevalencia de $1 / 143$ a $1 / 20000$ nacidos vivos. ${ }^{3,4,6}$

La última actualización en la clasificación de las IDP las divide en 9 categorías según el compartimiento del sistema inmune afectado. ${ }^{2}$

La presunción diagnóstica de las IDP se fundamenta en los 10 signos de alarma establecidos por Jeffrey Modell Foundation. ${ }^{7,8}$ Sin embargo, algunas inmunodeficiencias podrían presentar pocos o ningún signo de alarma.

El diagnóstico se establece según los criterios definidos por la European Society for Immunodeficiencies (ESID) y el Pan-American Group for Immunodeficiency (PAGID), que las divide en diagnóstico posible, probable o definitivo., ${ }^{9,10}$

A pesar del conocimiento molecular de estas patologías, muchos pacientes permanecen sin diagnóstico y/o son diagnosticados tardíamente, con consecuencias clínicas desfavorables. ${ }^{11}$

Los objetivos del presente trabajo son describir las características clínicas de pacientes con diagnóstico de IDP, analizar la presencia de signos de alarma y comparar las manifestaciones clínicas entre pacientes con déficit selectivo de IgA y otras IDP.

\section{POBLACIÓN Y MÉTODOS}

El Servicio de Alergia e Inmunología de la Clínica Universitaria Reina Fabiola tiene establecidos protocolos de estudio de pacientes con sospecha de IDP, de acuerdo con pautas internacionales. ${ }^{1}$ Los estudios varían según el compartimiento del sistema inmune afectado.

Se evaluó, en los pacientes, la presencia de los 
signos de alarma de IDP establecidos por Jeffrey Modell Foundation. ${ }^{7,8}$ Estos conforman una herramienta clínica que permite sospechar qué paciente tiene mayor probabilidad de padecer una IDP. Estos son: 4 o más otitis en un año; 2 o más sinusitis graves en un año; 2 meses o más de tratamiento antibiótico; 2 neumonías o más en un año; dificultad del crecimiento y desarrollo; abscesos profundos recurrentes; aftas persistentes en la boca o infecciones micóticas en la piel; necesidad de antibiótico endovenoso; 2 infecciones profundas o más (incluida la septicemia) y antecedentes familiares de IDP.

Se realizó un estudio retrospectivo, observacional y analítico.

Se incluyeron pacientes registrados en la base de datos de IDP del Servicio de Alergia e Inmunología con diagnóstico probable o definitivo de IDP9,10 (Tabla 1) y se los clasificó de acuerdo con las categorías establecidas en la última clasificación. ${ }^{2}$ Se los dividió en dos grupos: con déficit selectivo de IgA y con otras IDP.

Se excluyeron aquellos pacientes con diagnóstico posible de IDP y con inmunodeficiencias secundarias.

\section{Consideraciones éticas}

El estudio cumplió con la declaración de Helsinki y buenas prácticas clínicas de la Administración Nacional de Medicamentos, Alimentos y Tecnología Médica (ANMAT), la Ley Provincial de Investigación en Seres Humanos $\mathrm{N}^{\circ}$ 9694 y la Ley de Protección de Datos Personales $\mathrm{N}^{\circ}$ 25326. Fue aprobado por Comité Institucional de Ética.

\section{Análisis estadístico}

Se realizó un análisis descriptivo de las variables y las comparaciones mediante la prueba chi cuadrado de independencia para variables cualitativas y mediante la prueba de MannWhitney para variables cuantitativas. Se consideró estadísticamente significativo $\mathrm{p}<0,05$.

\section{RESULTADOS}

Se evaluaron 89 casos de IDP. Las características generales de los pacientes se observan en la Tabla 2.

Las principales manifestaciones clínicas de los pacientes fueron infección respiratoria alta y baja y enfermedades alérgicas (Figura 1). La infección por Candida se presentó en pacientes con alteraciones por disregulación inmunológica y con defectos de la inmunidad innata. Se registró un antecedente de internación en $37(41,6 \%)$ pacientes y $5(5,62 \%)$ fallecieron como consecuencia de su inmunodeficiencia.

Veinticuatro pacientes (26,97\%) presentaron signos de alarma de IDP. Estos fueron: 4 o más otitis en un año ( 5 pacientes), 2 o más sinusitis en un año (6 pacientes), más de dos meses de tratamiento antibiótico (9 pacientes), 2 neumonías o más en un año (14 pacientes), fallo de crecimiento (5 pacientes), abscesos cutáneos profundos (2 pacientes), lesiones orales persistentes (4 pacientes), uso de antibiótico endovenoso (15 pacientes), infecciones profundas (3 pacientes) y antecedentes familiares de IDP (9 pacientes).

Ninguno de los pacientes tenía antecedentes de consanguinidad.

TABLA 1. Criterios diagnósticos de inmunodeficiencias primarias (ESID-PAGID) ${ }^{9}$

\begin{tabular}{ll}
\hline Categoría de diagnóstico & \multicolumn{1}{c}{ Definición } \\
\hline Definitivo & Pacientes que tienen diagnóstico molecular-genético causal de la patología (falla genética, \\
& ausencia de ARNm o de la proteína responsable de la patología). \\
& Esta categoría tiene una probabilidad mayor del $98 \%$ de mantener el mismo diagnóstico \\
& en los próximos 20 años. \\
\hline Probable & Pacientes diagnosticados por fenotipo: características clínicas y/o de laboratorio \\
& compatibles con la patología, pero que no tienen identificada la falla genética responsable. \\
& Esta categoría tiene una probabilidad mayor del $85 \%$ de mantener el mismo diagnóstico \\
& en los próximos 20 años. \\
\hline Posible & Pacientes que tienen algunas pero no todas las características clínicas y de laboratorio \\
& compatibles con la patología. \\
\hline
\end{tabular}


TABLA 2. Características demográficas según grupo de inmunodeficiencias primarias ${ }^{* * *}$

\begin{tabular}{|c|c|c|c|c|c|c|c|}
\hline & $\mathbf{N}$ & $\begin{array}{c}\text { Criterio } \\
\text { diagnóstico } \\
\text { definitivo } \\
\end{array}$ & $\begin{array}{c}\text { Sexo } \\
\text { femenino } \\
(\%) \\
\end{array}$ & $\begin{array}{c}\text { Edad de } \\
\text { inicio de } \\
\text { síntomas (años) } \\
\end{array}$ & $\begin{array}{c}\text { Edad de } \\
\text { consulta } \\
\text { (años) }\end{array}$ & $\begin{array}{l}\text { Edad } \\
\text { de Dx } \\
\text { (años) } \\
\end{array}$ & $\begin{array}{c}\text { Intervalo entre } \\
\text { inicio de síntomas } \\
\text { y Dx (años) }\end{array}$ \\
\hline $\begin{array}{l}\text { Total de pacientes } \\
\text { con IDP }\end{array}$ & 89 & $59 / 89$ & $36 / 89$ & $3(1-5)^{* *}$ & $\begin{array}{c}6 \\
(4,08-11,67)^{* *}\end{array}$ & $\begin{array}{c}6 \\
(4,08-11,67)^{* *}\end{array}$ & $\begin{array}{c}2,83 \\
(1,08-5,83)^{* *}\end{array}$ \\
\hline ID combinadas & $1 / 89$ & $1 / 1$ & $1 / 1$ & 1,33 & 12 & 12 & 10,67 \\
\hline $\begin{array}{l}\text { Sme. bien definidos } \\
\text { con ID }\end{array}$ & $8 / 89$ & $2 / 8$ & $3 / 8$ & $\begin{array}{c}1 \\
(0,37-1,27)^{* *}\end{array}$ & $\begin{array}{c}5,04 \\
(2,37-6,5)^{* *}\end{array}$ & $\begin{array}{c}3,25 \\
(2,18-4)^{* *}\end{array}$ & $\begin{array}{c}2,13 \\
(1,31-2,85)^{* *}\end{array}$ \\
\hline $\begin{array}{l}\text { ID predominantemente } \\
\text { de anticuerpos* }\end{array}$ & $71 / 89$ & $50 / 71$ & $25 / 71$ & $\begin{array}{c}3 \\
(1,5-5)^{* *}\end{array}$ & $\begin{array}{c}6,08 \\
(4,33-11)^{* *}\end{array}$ & $\begin{array}{c}6,41 \\
(4,6-11,04)^{* *}\end{array}$ & $\begin{array}{c}3 \\
(1,04-5,79)^{* *}\end{array}$ \\
\hline $\begin{array}{l}\text { Enfermedades por } \\
\text { disrregulación inmune }\end{array}$ & $1 / 89$ & $1 / 1$ & $1 / 1$ & 2 & 2,83 & 4,67 & 2,67 \\
\hline Defecto de fagocitos & $1 / 89$ & $0 / 1$ & $1 / 1$ & 10 & 12,5 & 13 & 3 \\
\hline $\begin{array}{l}\text { Defectos de la } \\
\text { inmunidad innata }\end{array}$ & $2 / 89$ & $0 / 2$ & $2 / 2$ & $\begin{array}{c}1,25 \\
(0,87-0,95)^{* *}\end{array}$ & $\begin{array}{c}1,25 \\
(1,21-1,29)^{* *}\end{array}$ & $\begin{array}{c}2,83 \\
(2,25-3,41)^{* *}\end{array}$ & $\begin{array}{c}1,92 \\
(1,38-2,46)^{* *}\end{array}$ \\
\hline $\begin{array}{l}\text { Déficit de } \\
\text { complemento }\end{array}$ & $5 / 89$ & $5 / 5$ & $2 / 2$ & $\begin{array}{c}5 \\
(4-6)^{* *}\end{array}$ & $\begin{array}{c}36 \\
(15,42-50)^{* * *}\end{array}$ & $\begin{array}{c}36 \\
(15,42-50)^{* *}\end{array}$ & $\begin{array}{c}32 \\
(2-45)^{* *}\end{array}$ \\
\hline
\end{tabular}

ID: inmunodeficiencia; IDP: inmunodeficiencia primaria; Sme: síndrome; Dx: diagnóstico.

* 54/71 son pacientes con diagnóstico de déficit selectivo de IgA.

** Mediana (Q1-Q3).

*** No hubo pacientes con diagnósticos de IDP de las categorías enfermedades autoinflamatorias y fenocopias de IDP.

Figura 1. Manifestaciones clínicas de los pacientes con déficit selectivo de inmunoglobulina A y con otras inmunodeficiencias primarias

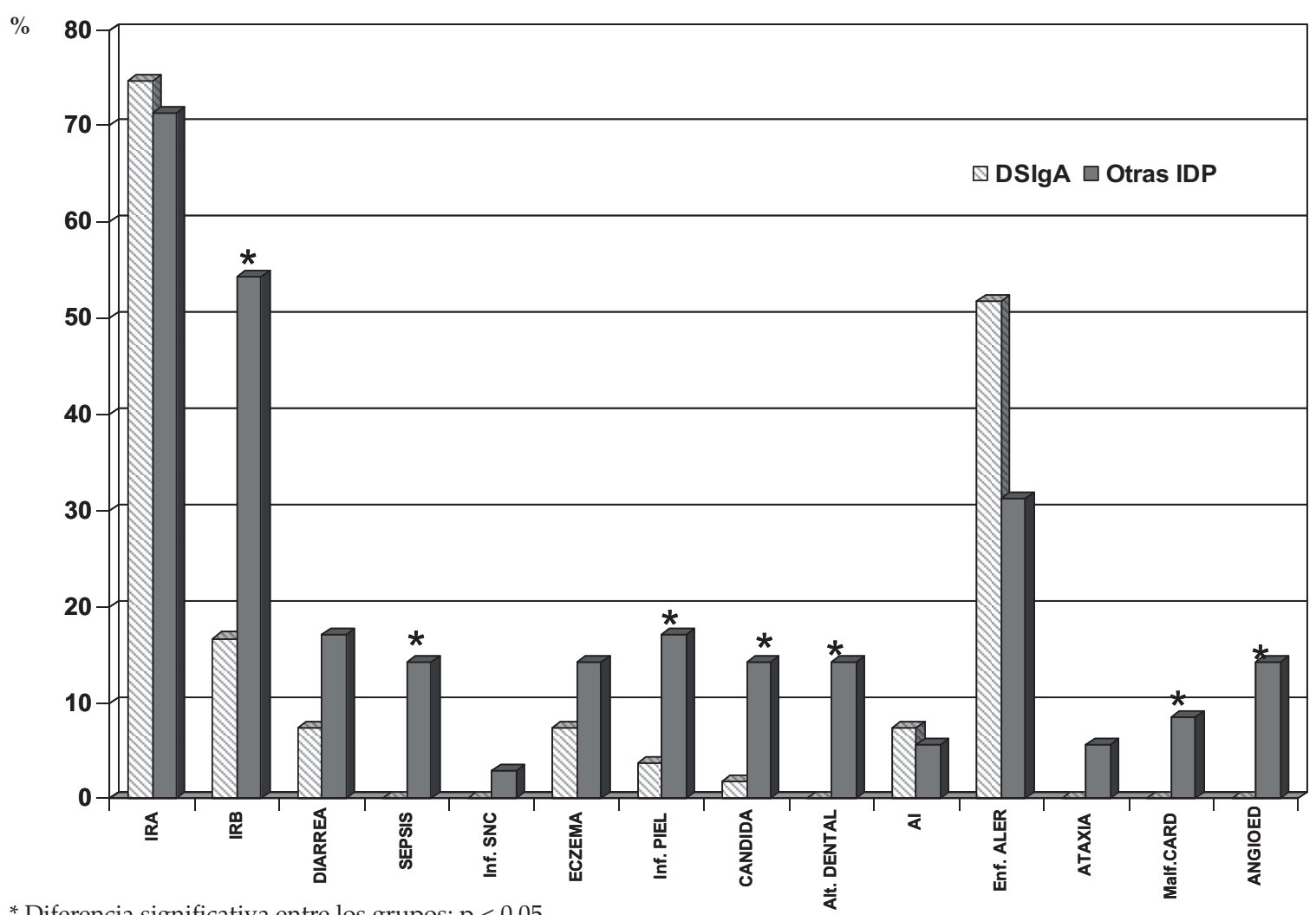

* Diferencia significativa entre los grupos: $\mathrm{p}<0,05$.

IRA: infección respiratoria alta; IRB: infección respiratoria baja; Inf. SNC: infección sistema nervioso central; AI: autoinmunidad; Enf ALER: enfermedad alérgica; Malf CARD: malformación cardiovascular; ANGIOED: angioedema;

DSIgA: déficit selectivo de IgA; IDP: inmunodeficiencia primaria. 
Se indicó tratamiento con gamaglobulina endovenosa a $10(11,24 \%)$ pacientes, los cuales pertenecían al grupo de IDP predominantemente de anticuerpos (agamaglobulinemia ligada al cromosoma $X$, inmunodeficiencia común variable y déficit de respuesta de anticuerpos); antibiótico-profilaxis a $16(17,98 \%)$ pacientes (deficiencia de STAT5b, ataxia-telangiectasia, síndrome de hiper-IgE, síndrome de Wiskott-Aldrich, agamaglobulinemia ligada al cromosoma $X$, inmunodeficiencia común variable, hipogamaglobulinemia transitoria de la infancia, deficiencia de subclase IgG2, déficit selectivo de IgA y enfermedad granulomatosa crónica); y antimicótico-profilaxis a dos $(2,24 \%)$ pacientes (enfermedad granulomatosa crónica e inmunodeficiencia común variable).

Se encontró un predominio del sexo masculino en ambos grupos; en el déficit selectivo de $\operatorname{IgA}$, $33(61,11 \%)$ pacientes; y en otras IDP, $20(57,14 \%)$ pacientes.

Las características generales de cada grupo se describen en la Tabla 3.

Las manifestaciones de enfermedad alérgica en el grupo con déficit selectivo de IgA fueron mayores que en el grupo de otras IDP (28 pacientes $-51,85 \%-$ y 11 pacientes $-31 \%-$ respectivamente), sin diferencia estadística significativa ( $\mathrm{p}=0,0578)$ (Figura 1).

La manifestación de patologías autoinmunes en nuestra población fue 4/54 (7,4\%) pacientes en el grupo con déficit selectivo de IgA y $2 / 35$ (5,7\%) pacientes en el grupo con otras IDP (Figura 1).

\section{DISCUSIÓN}

El diagnóstico temprano de las IDP permite un tratamiento precoz que mejora la calidad de vida y los costos de salud, por lo que la sospecha clínica del pediatra de cabecera es crítica en el diagnóstico y la derivación oportuna. ${ }^{12}$

El pronóstico de las IDP está asociado al diagnóstico precoz, que permite instaurar un tratamiento temprano, y a la enfermedad específica, que puede comprometer más gravemente al niño y condicionar su evolución. Uno de los pilares del diagnóstico son los signos de alarma establecidos por Jeffrey Modell Foundation, ${ }^{8}$ herramienta clave para la sospecha de estas patologías.

La mayoría de los diagnósticos de IDP son defectos predominantemente de anticuerpos. ${ }^{3,4,13}$ El déficit selectivo de IgA fue el más prevalente, ${ }^{14,15}$ al igual que en otras series.

A diferencia de lo encontrado en la bibliografía, se observó un elevado porcentaje de pacientes con antecedentes familiares positivos

TABLA 3. Características comparativas entre pacientes con déficit selectivo de inmunoglobulina A y pacientes con otras inmunodeficiencias primarias

\begin{tabular}{lccc}
\hline & $\begin{array}{c}\text { Déficit selectivo } \\
\text { de IgA (n: 54) }\end{array}$ & $\begin{array}{c}\text { Otras IDP } \\
\text { (n: 35) }\end{array}$ & P \\
\hline $\begin{array}{l}\text { Edad de inicio de los síntomas } \\
\text { (mediana - Q1-Q3) }\end{array}$ & $3,25(2-5)$ & $1,50(0,83-4)$ & 0,0167 \\
$\begin{array}{l}\text { Edad de 1) } \\
\text { (mediana - Q1-Q3) }\end{array}$ & $6,59(4,83-11)$ & $5,17(2,17-15,42)$ & 0,1670 \\
$\begin{array}{l}\text { Edad de diagnóstico } \\
\text { (mediana - Q1-Q3) }\end{array}$ & $6,88(5-11)$ & $4(2,58-15,42)$ & 0,0260 \\
$\begin{array}{l}\text { Tiempo entre inicio de síntomas y Dx } \\
\text { (mediana - Q1-Q3) }\end{array}$ & $3,50(1,08-5,75)$ & $2,42(1-6)$ & 0,8271 \\
SA+ (\%) & $0(0)$ & $24(68,57)$ & $<0,000$ \\
Cantidad de SA+ & & $2(0-3)$ & $<0,0001$ \\
(mediana - Q1-Q3) & $0(0-0)$ & $10(28,57)$ & $<0,000$ \\
Gamaglobulina EV (\%) & $0(0)$ & $15(42,85)$ & $<0,000$ \\
Antibiótico-profilaxis (\%) & $1(1,85)$ & $2(5,71)$ & 0,1519 \\
Antimicótico-profilaxis (\%) & $0(0)$ & $24(68,6)$ & $<0,000$ \\
Internaciones (\%) & $13(24,07)$ & $5(14,3)$ & 0,0043 \\
Pacientes fallecidos (\%) & $0(0)$ & & \\
\hline
\end{tabular}

IDP: inmunodeficiencias primarias; Dx: diagnóstico; SA: signos de alarma; IgA: inmunoglobulina A; EV: endovenosa. 
para IDP y ninguno de los pacientes incluidos presentó antecedentes de consanguinidad. ${ }^{4} \mathrm{La}$ ausencia de consanguinidad puede ser atribuida al tamaño muestral pequeño.

En aquellos pacientes con diagnóstico definitivo de IDP, se recomienda estudiar a su familia según el patrón de herencia que presente la patología diagnosticada.

La mediana de edad de inicio de los síntomas y de diagnóstico de la IDP fue menor a la encontrada en otros estudios. ${ }^{4,13}$

Al igual que otros autores, ${ }^{4}$ detectamos un ligero predominio del sexo masculino.

La mayoría de los pacientes con un déficit inmune profundo presentó signos de alarma de IDP, 7 mientras que ningún déficit selectivo de IgA presentó esta condición clínica, lo cual avala que el reconocimiento de los signos de alarma favorece un diagnóstico temprano de las IDP en la consulta pediátrica general.

La proporción de muertes por inmunodeficiencia fue menor a la descrita en otras series de pacientes. ${ }^{4}$

La infección por Candida sp., es una manifestación clínica generalmente asociada a defectos combinados y de los fagocitos; ${ }^{1}$ a diferencia de esto, en nuestra investigación, se presentó en pacientes con alteraciones por disregulación inmunológica y con defectos de la inmunidad innata.

La proporción de enfermedad alérgica en pacientes con déficit selectivo de IgA fue mayor que en el grupo con otras IDP, lo cual coincide con la literatura, en la que la atopia es la segunda manifestación clínica luego de las infecciones recurrentes en estos pacientes. ${ }^{14,15}$

La prevalencia de enfermedades autoinmunes observada fue menor que la informada en otros trabajos de investigación. ${ }^{14}$

El tamaño muestral de este estudio y su diseño retrospectivo son condiciones limitantes para sacar conclusiones definitivas con respecto a las patologías descritas.

\section{CONCLUSIONES}

La principal manifestación clínica de los pacientes con IDP fueron las infecciones respiratorias. La enfermedad alérgica fue una manifestación clínica frecuente en los pacientes con déficit selectivo de IgA.

Los signos de alarma estuvieron presentes en las IDP graves.

La complejidad del tratamiento de los pacientes con IDP graves fue mayor que en los pacientes con déficit selectivo de IgA.

\section{Agradecimientos}

A la Téc. Eugenia Concari, al Dr. Arnaldo Mangeaud y a la Dra. Marina F. Ponzio por su colaboración en la elaboración de este trabajo de investigación.

\section{REFERENCIAS}

1. Notarangelo LD. Primary immunodeficiencies. J Allergy Clin Immunol 2010;125(2 Suppl 2):S182-94.

2. Al-HerzW, Bousfiha A, CasanovaJL, Chatila T, etal.Primary immunodeficiency diseases: an update on the classification from the International Union of Immunological Societies Expert Committee for Primary Immunodeficiency. Front Immunol 2014;5:162.

3. Grupo de Inmunología Pediátrica. Registro argentino de inmunodeficiencias primarias. Segundo informe. Arch Argent Pediatr 2007;105(5):453-60.

4. Gathmann B, Binder N, Ehl S, Kindle G. The European internet-based patient and research database for primary immunodeficiencies: update 2011. Clin Exp Immunol 2012;167(3):479-91.

5. De Vries E. Patient-centred screening for primary immunodeficiency: a multi-stage diagnostic protocol designed for non-immunologists. Clin Exp Immunol 2006;145(2):204-14.

6. Aghamohammadi A, Abolhassani H, Biglari M, Abolmaali $\mathrm{S}$, etal. Analysis of switched memory B cells in patients with IgA deficiency. Int Arch Allergy Immunol 2011;156(4):462-8.

7. Leiva LE, Bezrodnik L, Oleastro M, Condino-Neto A, et al. Primary immunodeficiency diseases in Latin America: proceedings of the second Latin American Society for immunodeficiencies (LASID) Advisory Board. Allergol Immunopathol (Madr) 2011;39(2):106-10.

8. Jeffrey Modell Foundation. 10 warning signs. New York: Jeffrey Modell Foundation; 2013. Disponible en: http:/ / www.info4pi.org/library/educational-materials /10warning-signs. [Acceso: 16 de mayo de 2014].

9. Conley ME, Notarangelo LD, Etzioni A. Diagnostic criteria for primary immunodeficiencies. Representing PAGID (Pan-American Group for Immunodeficiency) and ESID (European Society for Immunodeficiencies). Clin Immunol 1999;93(3):190-7.

10. Ehl S. New clinical diagnosis criteria for the ESID Registry. Geneva: European Society for Immunodeficiencies; 2014. Disponible en: http://esid.org/Working-Parties/ Registry / Diagnosis-criteria. [Acceso: 5 de marzo de 2014].

11. Ballow M, Notarangelo L, Grimbacher B, CunninghamRundles C, et al. Immunodeficiencies. Clin Exp Immunol 2009;158(Suppl 1):14-22.

12. Costa-Carvalho BT, Grumach AS, Franco JL, EspinosaRosales FJ, et al. Attending to warning signs of primary immunodeficiency diseases across the range of clinical practice. J Clin Immunol 2014;34(1):10-22.

13. Joshi AY, Iyer VN, Hagan JB, St Sauver JL, et al. Incidence and temporal trends of primary immunodeficiency: a population-based cohort study. Mayo Clin Proc 2009;84(1):16-22.

14. Domínguez O, Giner MT, Alsina L, Martín MA, et al. Fenotipos clínicos asociados a la deficiencia selectiva de IgA: revisión de 330 casos y propuesta de un protocolo de seguimiento. An Pediatr (Barc) 2012;76(5):261-7.

15. Yel L. Selective IgA deficiency. J Clin Immunol 2010;30(1):10-6. 